\title{
INDICADORES PARA MEDIR SITUACIONES DE VULNERALIDAD SOCIAL. PROPUESTA REALIZADA EN EL MARCO DE UN PROYECTO EUROPEO' ${ }^{1}$
}

\author{
SusAna R. NAVARRo RodRÍGUEZ \\ REMEDIOS LARRUBia VARGAS
}

\begin{abstract}
RESUMEN
A la hora de analizar la realidad social, especialmente las situaciones de vulnerabilidad, se han utilizado diversos enfoques y técnicas que han suscitado intensos debates sobre su idoneidad. Sin que exista un acuerdo unánime en la comunidad científica a la hora de establecer cual es el método más adecuado, sí se aprecia cierta decantación hacia los sistemas de indicadores sociales. En este artículo se presenta la trayectoria y características de esta técnica, así como el sistema de indicadores sociales para la inclusión social propuesto y aplicado en el marco de un proyecto europeo.
\end{abstract}

\section{ABSTRACT}

When analyzing the social reality, especially the vulnerability situations, diverse focuses have been used and technical that have raised intense debotes on their suitability. Without an unanimous agreement exists in the scientific community when to settle down which is the most appropriate method, yes certain decantation is appreciated toward the systems of social indicators. In this article the trajectory is presented and characteristic of this technique, as well as the system of social indicators to take a short cut the proposed social exclusion and applied in the mark of an European project.

1. Este artículo forma parte de un trabajo realizado para el área de bienestar social del Ayuntamiento de Málaga, dentro del marco del Proyecto Urbal-10, proyecto aprobado y subvencionado por la Comisión Europea. El proyecto ha contado con los siguientes socios: Aserrí de costa Rica, Feira de Santana de Brasil, Lima y Oyon de Perú, Navolato de Méjico, El Bosque de Chile, I.M.F.E. de Granada, España y F.I.D.D.E.M (con sede en St. Denis, Francia) bajo la coordinación del Ayuntamiento de Málaga. Uno de los objetivos del Proyecto Urbal es promover el intercambio de experiencias y difusión de buenas prácticas en la elaboración de instrumentos de planificación y evaluación de políticas sociales mediante el uso de un sistema de información geográfica que permita el análisis de indicadores sociales y su aplicación a un territorio, siendo la temática en torno a la que giraría la "Inclusión social" 


\section{INTRODUCCIÓN}

El interés por evaluar la realidad social a través de algún instrumento que permitiera su medición y cuantificación llevó a la configuración de los indicadores sociales, una técnica cuantitativa relativamente reciente que tiene la particularidad de medir realidades complejas y sintetizarlas hasta el punto de ser capaz de representarlas en un solo número o índice general.

La noción de indicador social se suele atribuir a Bauer cuando en el año 1966 publicó su libro sobre indicadores sociales, a partir de este momento surgieron relevantes estudios y publicaciones calificándose de "Movimiento de los Indicadores Sociales" ante el importante auge que había tomado, aunque los orígenes más remotos de los indicadores sociales se sitúan en los EEUU hacia 1930.

Los indicadores sociales se plantean, en un principio, como instrumento de medición para determinadas magnitudes económicas y sociales que permitieran el estudio de los comportamientos de éstas a lo largo del tiempo. A partir de su desarrollo posterior serán usados, en algunos casos, para el análisis de la satisfacción de determinadas necesidades, o al menos de la distribución de ciertos recursos que no son fácilmente evaluables de manera directa. La investigación social aplicada ha encontrado un apoyo importante en esta técnica a la hora de planificar, ejecutar y evaluar políticas sociales.

\section{TÉCNICAS PARA EL ESTUDIO DE LA VULNERABILIDAD SOCIAL. LOS INDICADORES SOCIALES}

\subsection{Características de los sistemas de indicadores sociales}

Desde que Bauer creó la expresión de indicador social se han sucedido múltiples definiciones al respecto, sin que se haya conseguido una definición exacta, rigurosa y consensuada del término. Se puede decir que su legitimidad, su objetividad se ha hecho en función de las finalidades más o menos perseguidas por los usuarios de los indicadores sociales. Los intentos de definición de un indicador social son muchos tanto desde el marco científico como institucional. La mayoría de estas definiciones o esbozos del concepto de indicador social recogen unos elementos reiterativamente tanto en el marco académico como operativo.

Los elementos que reiteradamente aparecen en el conjunto de las definiciones de indicadores sociales y que sirven para definirlos son los siguientes: 
- Medida estadística. Para que un indicador social exista se necesita que haya evaluación de una cantidad.

- Concepto, dimensión, aspecto, etc. Se trata de evaluar un concepto, una idea, un conjunto de fenómenos simples y directamente observables (pobreza, marginalidad).

- Análisis teórico previo. Este aspecto es lo que puede distinguir el indicador social de la simple estadística. La diferencia radica en que la construcción de indicadores es el resultado de un gran esfuerzo de análisis y de investigación en un campo. Por tanto el criterio que permite distinguir entre estadísticas e indicadores sociales es su valor informativo, basado en la reflexión teórica y en el desarrollo de un esquema explicativo.

- Integrado en un sistema coherente de medidas. Un indicador social no se concibe aisladamente, forma parte de un conjunto de medias interdependientes. Por lo tanto los indicadores sociales toman sentido al considerarlos como "Sistema", como construcciones ordenadas y coherentes.

- Describe el estado de la realidad social y en consecuencia de las políticas sociales. El describir el estado de la sociedad conlleva dos aspectos, el estado estático (actual o instantáneo) y el dinámico (su evolución).

Al igual que ocurre con la definición de los indicadores sociales, establecer una tipología constituye un importante reto debido a la confusión existente en la investigación y a la proliferación de las mismas sin que ninguna haya alcanzado el éxito adecuado(Carmona Guillén, J.A. 1977, 49-51). Un claro ejemplo de ello lo podemos apreciar en el cuadro 1 donde se sintetizan los diferentes criterios que se han utilizado para hacer una clasificación de indicadores sociales y las tipologías a las que han dado lugar.

\section{CUADRO 1 \\ TIPOLOGÍAS DE INDICADORES SOCIALES}

\begin{tabular}{|l|l|}
\hline \multicolumn{1}{|c|}{ CRITERIOS DE CLASIFICACIÓN } & \multicolumn{1}{c|}{ CLASIFICACIÓN } \\
\hline - POR LA ESCALA DE MEDIDA & $\begin{array}{l}\text { * Indicadores absolutos;* Indicadores } \\
\text { relativo }\end{array}$ \\
\hline - POR LA NATURALEZA DEL HECHO & $\begin{array}{l}\text { * Datos de observación objetiva; Fenómenos } \\
\text { percibidos o subjetivos }\end{array}$ \\
\hline - POR EL NIVEL DE ABSTRACCIÓN & $\begin{array}{l}\text { * Indicadores simples;* Indicadores } \\
\text { sintéticos }\end{array}$ \\
\hline - POR EL CONTENIDO TEÓRICO & $\begin{array}{l}\text { * Indicadores analíticos; * Indicadores } \\
\text { descriptivos }\end{array}$ \\
\hline
\end{tabular}




\begin{tabular}{|l|l|}
\hline \multicolumn{1}{|c|}{ CRITERIOS DE CLASIFICACIÓN } & \multicolumn{1}{c|}{ CLASIFICACIÓN } \\
\hline - POR LA TEMÁTICA DE ESTUDIO & $\begin{array}{l}\text { * Indicadores económicos; Indicadores de } \\
\text { calidad de vida; Indicadores de exclusión } \\
\text { social, etc }\end{array}$ \\
\hline $\begin{array}{l}\text { - POR LOS MEDIOS Y LOS RESULTADOS } \\
\text { OBTENIDOS }\end{array}$ & $\begin{array}{l}\text { * Indicadores internos; Indicadores externos; } \\
\text { Indicadores de medio; Indicadores de } \\
\text { producto }\end{array}$ \\
\hline - POR SU UBICACIÓN EN UN MODELO \\
INTERNACIONAL & $\begin{array}{l}\text { * Indicadores de insumo; Indicadores de } \\
\text { proceso; Indicadores de efecto o resultado; } \\
\text { Indicadores de impacto; Indicadores } \\
\text { de eficacia; Indicadores de suficiencia; } \\
\text { Indicadores de acceso. }\end{array}$ \\
\hline
\end{tabular}

Para realizar un sistema de indicadores sobre hechos sociales en los que recae nuestra atención y que queremos analizar, el primer problema que encontramos es que los conceptos muy amplios son difícilmente medibles por lo que las distintas organizaciones que han intentado afrontar el reto de realizar sistemas de indicadores sociales siempre indican la metodología usada de las que, al igual que en el caso del concepto de indicador social, se pueden extraer hitos comunes.

La línea metodológica propuesta por el Consejo Económico y Social de las Naciones Unidas ha sido una de las más utilizadas en los organismos internacionales especializados en el tema (OCDE, ONU) (Zarzosa Espina, P. 1996). Los principales pasos que propone para realizar un sistema de indicadores sociales son los siguientes:

- En primer lugar se trataría de descomponer el objetivo a medir (concepto) en áreas, componentes, campos, preocupaciones sociales, etc, dependiendo de la nomenclatura utilizada en las distintas metodologías.

- En segundo lugar estas dimensiones pueden dividirse en subdimensiones o subcomponentes que siguen siendo variables teóricas susceptibles de medirse con indicadores.

- En tercer lugar, una vez hecha esta división, se trata de encontrar, para cada componente, o subdimensión una o varias medidas que indiquen su estado.

Una vez elegida la metodología se pasa a la selección de los indicadores en función de un criterio. Los criterios para elaborar una primera lista de indicadores son numerosos si bien en todos ellos, al igual que ocurría en aspectos considerados anteriormente, podemos encontrar unas características comunes que nos pueden servir al final para realizar una propuesta definitiva de las características que debe tener un sistema de indicadores sociales: 
- OBJETIVIDAD Y SENCILLEZ. Una medición objetiva del problema, no muy costosa en su obtención y fácil de interpretar.

- ESPECIFICIDAD. Una medición directa del objeto, depurada de otras significaciones

- SENSIBILIDAD. Una medida cuyos valores sean sensibles a los cambios, progresión-involución o intensificación-atenuación del objeto.

\subsection{Sistemas de indicadores sociales nacionales e internacionales}

Los primeros intentos por desarrollar sistemas de estadísticas sociales comparables con los existentes en lo económico fueron llevados a cabo en los años 60 e inicios de los 70 por grupos académicos y científicos sociales en los Estados Unidos y Europa. Estos esfuerzos iniciales fueron seguidos por gestiones a nivel internacional de organizaciones como la OCDE (Organización para la Cooperación y el Desarrollo Económico) y las Naciones Unidas.

La primera propuesta de elaboración de los indicadores sociales por la OCDE fue promovida por los gobiernos de los países miembros de la organización en 1970.

El sistema de indicadores sociales de la OCDE tiene como finalidad manifiesta la medición del bienestar social. La metodología de la OCDE es la que hemos señalado con anterioridad, se trata de dividir el bienestar social en una serie de áreas o "campo de objetivos", esto, a su vez, se van dividiendo en subpreocupaciones y así sucesivamente, y, por último se elabora una lista de los indicadores sociales.

Tras la primera propuesta, que ha servido de base para todas las propuestas posteriores, en los primeros años del presente siglo la OCDE ha realizado un nuevo sistema de indicadores sociales. El objetivo esencial de esta nueva propuesta de indicadores es responder a dos cuestiones, una en relación a la evolución social llevada a cabo en los países miembros de la OCDE, y otra determinar los resultados sociales obtenidos de las acciones llevadas a cabo por la sociedad en general y por los gobiernos en concreto (Cuadro 2) (OCDE, 2002) 


\section{CUADRO 2 SISTEMA ACTUAL DE INDICADORES DE LA OCDE}

\begin{tabular}{|c|c|}
\hline Indicadores de contexto & $\begin{array}{l}\text { G1. Ingresos nacionales, G2. Índices de fertilidad, G3. Ni- } \\
\text { vel de dependencia de las personas mayores, G4. Extran- } \\
\text { jeros y población de origen extranjero, G5. Refugiados y } \\
\text { solicitantes de asilo, G6. Índices de divorcios, G7. Familias } \\
\text { monoparentales }\end{array}$ \\
\hline Indicadores de autosuficiencia & $\begin{array}{l}\text { Estado social/ Respuestas sociales, A1. Empleo/ A7. Políti- } \\
\text { cas de activación, A2. Paro /A8. Gastos en educación, A3. } \\
\text { Jóvenes sin empleo/ A9. Educación y cuidado infantiles a } \\
\text { una edad temprana, A4. Hogares sin empleo / A10. Logros } \\
\text { en la educación, A5. Madres trabajadoras /A11. Alfabetiza- } \\
\text { ción, A6. Edad de jubilación /A12. Índices de sustitución, } \\
\text { A13. Presión fiscal, B6. Gasto público social, B7. Gasto } \\
\text { público privado,B8. Gasto neto social,C6. Gente mayor en } \\
\text { residencias }\end{array}$ \\
\hline Indicadores de equidad & $\begin{array}{l}\text { Estado social /Respuestas sociales, B1. Pobreza relativa / } \\
\text { B5. Salarios mínimos } \\
\text { B2. Desigualdad en los ingresos /B6. Gasto público social, } \\
\text { B3. Empleo poco remunerado /B7. Gasto privado social, } \\
\text { B4. Diferencia salarial por razón de sexo/ B8. Gasto neto } \\
\text { social, A2. Paro /B9. Obtención de prestaciones, A3. Jóve- } \\
\text { nes sin empleo A7. Políticas de activación, A4. Hogares sin } \\
\text { empleo A8. Gastos en educación, A5. Madres trabajadoras } \\
\text { A9. Educación y cuidado infantiles a una edad temprana, } \\
\text { A10. Logros en la educación, A11. Alfabetización,A12. Ín- } \\
\text { dices de sustitución }\end{array}$ \\
\hline Indicadores de salud & $\begin{array}{l}\text { Estado social Respuestas societales, C1. Esperanza de vida } \\
\text { /C6. Personas mayores en residencias, C2. Mortalidad in- } \\
\text { fantil /C7. Gasto en asistencia médica } \\
\text { C3. Años potenciales de vida perdida /C8. Responsabilidad } \\
\text { para financiar la asistencia médica, C4. Esperanza de vida } \\
\text { sin padecer ninguna discapacidad /C9. Infraestructura mé- } \\
\text { dica, C5. Accidentes /A9. Educación y cuidado infantiles a } \\
\text { una edad temprana,B1. Pobreza relativa /A10. Logros en la } \\
\text { educación,A2. Paro } \\
\text { D2. Consumo de drogas y muertes relacionadas }\end{array}$ \\
\hline Indicadores de cohesión social & $\begin{array}{l}\text { Estado social /Respuestas societales, D1. Huelgas /D7 } \\
\text { Prisioneros,D2 Consumo de drogas y muertes relaciona- } \\
\text { das/ A6. Edad de jubilación, D3 Suicidio /A9 Educación y } \\
\text { cuidado infantiles a una edad temprana,D4. Crimen /A10 } \\
\text { Logros en la educación,D5. Pertenencia a un grupo /B6. } \\
\text { Gasto público social, D6. Votación /C7. Gasto en salud,B1 } \\
\text { Pobreza relativa,A2 Paro }\end{array}$ \\
\hline
\end{tabular}


En cuanto a la $\mathbf{O N U}$, el comienzo de los trabajos sobre indicadores sociales hay que situarlo en un informe de 1960 en el que se recomendaba la elaboración de indicadores que permitiesen realizar comparaciones internacionales de los distintos componentes del nivel de vida y en el que se establecía una metodología básica en la que habría que asentarse el movimiento de indicadores sociales a punto de emerger.

$\mathrm{Al}$ igual que ocurriera con la OCDE, las Naciones Unidas también en los primeros años del S. XXI ha elaborado una segunda propuesta que es la que actualmente está en vigor, con cinco temas de políticas y áreas principales de incumbencia social surgidos de una serie de eventos mundiales (Cuadro 3).

\section{CUADRO 3 \\ PROPUESTA ACTUAL DE INDICADORES SOCIALES DE LA ONU}

\begin{tabular}{|l|l|}
\hline CAMPOS & DIMENSIONES \\
\hline 1. - POBLACIÓN Y DESARROLLO & $\begin{array}{l}\text { a.- Salud, b.- Bienestar material, c.- } \\
\text { Educación }\end{array}$ \\
\hline 2.- ERRADICACIÓN POBREZA & a. - Ingreso y gasto,b. - Recursos económicos \\
\hline $\begin{array}{l}\text { 3.- EXPANSIÓN DEL EMPLEO } \\
\text { PRODUCTIVO/REDUCCIÓN } \\
\text { DESEMPLEO }\end{array}$ & $\begin{array}{l}\text { a.- Trabajo, b. - Ambiente laboral, c. - } \\
\text { Educación y entrenamiento }\end{array}$ \\
\hline 4.- INTEGRACIÓN SOCIAL & $\begin{array}{l}\text { a. - Vivienda, b. - Trabajo, c. - Crimen y } \\
\text { justicia criminal }\end{array}$ \\
\hline 5.- ESTADO DE MUJERES Y HOMBRES & $\begin{array}{l}\text { a. - Saludos cordiales, educación, b. } \\
\text { - Trabajo, c. - Ingresos }\end{array}$ \\
\hline
\end{tabular}

A partir de los trabajos sobre los indicadores sociales de los dos organismos citados, se iniciaron múltiples y variados programas en la mayoría de los países desarrollados. A la cabeza de estas experiencias se sitúa Estados Unidos, con un informe de 1969, seguido en 1970 por el Reino Unido. Durante toda la década de los 70 van surgiendo informes sobre indicadores sociales, en prácticamente todos los países, así como en otros organismos internacionales, como la actual UE que abre su programa de indicadores sociales en 1975, programa cuya segunda etapa se puso en marcha en 1991, "Retrato social de Europa".

Bajo el paraguas de todos estos intentos regionales se sitúan los españoles, cuyo inicio es el año 1975, con la aparición de "España: Panorámica Social" del INE que culmina con la más reciente publicación de los Indicadores sociales (1991).

El objetivo expreso del proyecto del INE es la medición del bienestar. Y de forma análoga como se hace en los proyectos de la ONU y la OCDE se agrupa los temas objetos de investigación en ocho "Campos de preocupación 
social”: Educación, Trabajo, Distribución y consumo, Protección y servicios sociales, Salud, Vivienda y medio ambiente, Cultura y ocio, Oportunidades sociales y participación. Además se prevé la inclusión de tres capítulos de indicadores adicionales: Población, Familias y hogares, Investigación y Tecnología

En el caso de la Comunidad Andaluza la elaboración del Sistema de Indicadores sociales ha sido obra del Instituto de Estadística de Andalucía (2005), con el objetivo de proporcionar unos datos empíricos que permitan la evaluación de la calidad de vida en esta Comunidad mediante el seguimiento en el tiempo de los cambios experimentados por la estructura social. Las Áreas y Dimensiones seleccionadas han sido 8: Población, Entorno físico y social, Educación, Trabajo, Riqueza, renta y consumo, Cultura y ocio, Salud y Participación, que han dado lugar a 44 dimensiones.

Por último, no podemos dejar de mencionar el sistema de indicadores sociales que desde el Ministerio de Trabajo y Asuntos Sociales se ha elaborado para el seguimiento de los Planes Nacionales de Acción para Inclusión social desarrollados a partir del año 2001. Los indicadores se organizan en 12 ámbitos cada uno de ellos con sus respectivos indicadores. Estos ámbitos contemplan diferentes realidades (empleo, vivienda, educación, salud, familia, población discapacitada, inmigrante, gitana etc) que son indicativas de vulnerabilidad social y por lo tanto requieren poner en marcha programas de inclusión social, además también incluyen indicadores de gestión.

\section{PLANES Y ACCIONES PARA LA INCLUSIÓN SOCIAL}

\subsection{Significado de la exclusión social}

El término exclusión social se comenzó a utilizar durante los años setenta en Francia, aunque no se generalizó en el lenguaje de las políticas públicas y de las ciencias sociales hasta las décadas de los ochenta y los noventa. En aquel momento, la exclusión social quedó asociada sobre todo al concepto de desempleo y a la inestabilidad de los vínculos sociales, empezando a desarrollarse algunas políticas específicas para su «reinserción». Posteriormente surgieron iniciativas y propuestas para superar este concepto insuficiente de pobreza.

En esta nueva línea se considera que la exclusión social como un fenómeno conceptualmente más amplio que la pobreza, al menos cuando se entiende ésta última en términos de nivel de vida material, siguiendo el enfoque más convencional. Aunque la exclusión social y la pobreza tienen amplias zonas de intersección, no son conceptos sinónimos ni necesariamente coincidentes. 
Pero conviene no perder de vista el papel fundamental que la pobreza tiene en los procesos de exclusión social, así como el hecho de que la pobreza es, casi siempre, la forma a través de la cual se manifiesta la exclusión. También la marginación implica exclusión pues al estar un sistema al margen o alejado de los comportamientos y valores sociales predominantes tiene como consecuencia, en muchos casos, verse privado de los derechos y beneficios económicos, sociales y culturales.

La relación entre la pobreza y la marginación no es unidireccional. Los procesos de marginación suelen estar asociados y reforzados por situaciones de carencia o pobreza económica, pero la pobreza no conduce necesariamente a la marginación. Por otro lado, la marginación puede afectar a grupos sin carencias económicas, como es el caso de algunas enfermedades crónicas como el VIH o las enfermedades mentales crónicas-, algunas etnias, minorías inmigrantes, $\mathrm{u}$ otros, hundiendo sus raíces en factores de orden psicológico, individual y sociorelacional, que unidos a las carencias del orden social o institucional acaban socavando los recursos económicos, las capacidades de inserción laboral y los apoyos sociales y afectivos de los individuos -como es el caso de algunas toxicomanías-.

Actualmente, nuevos mecanismos de segregación social han ido tomando cuerpo y se consideran significativos a la hora de impedir o dificultar a las personas su acceso al mercado de trabajo, a la vivienda, la formación, etc.: la llamada fragmentación de la sociedad que hace de la nueva realidad un cuerpo social mucho más complejo y diverso.

Estas situaciones pueden verse reforzadas por el significativo déficit de las políticas públicas que se habían ido implantando en décadas anteriores en toda Europa, y más tardíamente en España, y por las dificultades para responder a todo ello desde la iniciativa social.

De este modo, en su manifestación compleja y actual, la exclusión social es un concepto integral, que puede tomar forma en cualquiera de los ámbitos vitales básicos de las personas, con una gran variedad y gravedad de las situaciones que desencadena o sostiene, por lo que la perspectiva más adecuada para su estudio debe ser flexible, dinámica y que tenga en cuenta todas las dimensiones o ámbitos: el económico, el laboral, el formativo, el sociosanitario, el residencial, el relacional y el ámbito de la ciudadanía y la participación.

La lucha contra la exclusión social a nivel comunitario tuvo su punto de arranque en el Consejo europeo de Lisboa y Feira del 2000, y se ha materializado por un lado en la aprobación de la iniciativa que puso en marcha los Planes Nacionales de Acción que fueron progresivamente acomodándose al método abierto de coordinación con que el que viene trabajando la UE, y por otro en mejorar los indicadores en el ámbito de la pobreza y la exclusión social. En Lisboa no sólo se sientan las bases de la lucha europea contra la 
exclusión social sino que también se avanza en el compromiso de los Estados miembros en materia de inclusión social.

Dado que los Planes Nacionales de Acción elaborados en 2001 tenían una vigencia de dos años, a finales de 2002 se inician las actuaciones preparatorias para la puesta en marcha de la segunda ronda de Planes Nacionales de Acción (2003-2005), también se aprueba la necesidad de reforzar la perspectiva de género y los riesgos de pobreza y exclusión social que afectan a los inmigrantes y de emitió un documento marco en el que se establecían las directrices comunes de los Planes Nacionales de Acción para 2003-2005.

Por lo que respecta a los indicadores en el ámbito de la pobreza y la exclusión social los Estados miembros y la Comisión han tratado de perfilar planteamientos comunes de cara a la definición de indicadores y de garantizar la compatibilidad de los mismos.

Se consideró necesario disponer de un gran número de indicadores para evaluar correctamente la naturaleza pluridimensional de la exclusión social, proponiendo el Comité de protección social una clasificación por orden de prioridad: los indicadores primarios que cubrirían aquellos ámbitos considerados más importantes de cara a la exclusión social (renta, cohesión regional, desempleo, esperanza de vida, estado de salud y situación respecto a la enseñanza y los indicadores secundarios (persistencia de renta baja, desempleo y niveles educativos) que describirían otras dimensiones del problema, serían definidos y adoptados de común acuerdo y serían utilizados por los Estados miembros para la elaboración de sus respectivos planes nacionales de acción sobre la inclusión social.

\section{EL SISTEMA DE INDICADORES SOCIALES EN EL MARCO DEL PROYECTO URBAL}

\subsection{Aspectos fundamentales para avanzar en políticas de inclusión social desde una perspectiva territorial}

Nuestro objetivo es establecer un sistema de indicadores que nos permita conocer la vulnerabilidad social de los individuos que habitan en las distintas áreas de las ciudades que integran el proyecto, y de los espacios que se encuentran en mayor riesgo de exclusión social.

Nuestra formulación se basa en la utilización de indicadores cuantitativos y simples suministrados por las fuentes estadísticas, ya que buscamos indicadores que podamos obtener de forma sencilla y renovable, no sujetos al encarecimiento y dificultad de obtención que plantea la utilización de encuestas y entrevistas para aproximarnos a la realidad social, aunque la información 
obtenida de este modo somos conscientes que posee un innegable valor, y que en ocasiones resulta imprescindible.

Esta opción sin embargo no elimina las dificultades a la hora de hacer la selección de variables debido a las carencias que presentan las fuentes estadísticas oficiales. Una problemática que se agudiza al trabajar como es nuestro caso en el ámbito local, es decir en el análisis microespacial, ya que las fuentes más documentadas y completas ofrecen información agregada a nivel nacional y como mucho a nivel provincial.

Esto nos va a llevar cuando identifiquemos las dimensiones del fenómeno exclusión social, a que diseñemos en primera instancia como posibles indicadores de las mismas a un número amplio de variables estadísticas, que posteriormente en la propuesta final se depurarán en función de las posibilidades de su obtención.

Dos variables claves para poder realizar un estudio a nivel de SIG de cualquier aspecto y a cualquier escala de la población son la fecha de nacimiento de la población, que nos ofrecerá una información viva sobre la edad de la misma, junto al sexo de la misma, unos datos que podríamos denominar de contexto, y que son de vital importancia para conocer aspectos como la estructura de edad y sexo (índices de Juventud, envejecimiento, sex ratio ) y sobre la dinámica natural de la población (tasas de fecundidad, mortalidad, etc.).

Pero además como el fenómeno a analizar es la exclusión social, tras el estudio del marco teórico, creemos que las dimensiones en las que hay que trabajar serían: 1. Pobreza/Nivel de ingresos; 2. Trabajo; 3. Formación; 4. Salud; 5.Vivienda y entorno; 6. Vulnerabilidad/Precariedad familiar

Aunque podríamos añadir igualmente una dimensión que denominaríamos Participación ciudadana, en la que se conocería el grado de implicación social, como formar parte de organizaciones ciudadanas, o el grado de conocimiento y acceso a programas sociales.

\subsubsection{Pobreza}

En el análisis de la pobreza y en el de la desigualdad social internacionalmente se viene considerando esencial el indicador de carácter económico relativo a la renta per cápita. Todos los países elaboran por ello medidas relativas a la renta de sus poblaciones. Ahora bien, las estadísticas públicas sobre la renta y su estratificación se refieren a colectivos amplios de nivel nacional o regional.

Para ordenar a las familias según su posición económica e identificar a los que se sitúan por debajo del umbral, se suele utilizar la renta monetaria disponible, una variable que recoge fundamentalmente los ingresos que las familias obtienen de cualquier fuente (trabajo, capital y propiedad, transferen- 
cias públicas o privadas), tras el pago de impuestos directos y las cotizaciones obligatorias a la Seguridad Social satisfechos.

La forma más habitual de obtener la información sobre los ingresos de los hogares es a través de encuestas, como es en España la "Encuesta Continua de los Presupuestos Familiares" o el Panel de Hogares de la UE, e inclusive las organizadas por los propios investigadores como los estudios publicados por la fundación FOESSA y EDIS-Caritas que se basan en entrevistas directas a muestras representativas de la población.

Esta metodología presenta errores de medición característicos como la subestimación de los ingresos, en relación con los datos de fuentes externas como la Contabilidad nacional, o la falta de representación de los colectivos más marginados, pero además por su carácter de muestra, tampoco es adecuada para ser explotada en microanálisis social, ni en microanálisis territorial.

Algunos expertos defienden que el gasto realizado por las familias en la compra de bienes de consumo es una variable más adecuada que la renta corriente para estudiar la pobreza, porque los datos obtenidos a través de encuestas suelen ser más fiables que los datos de los ingresos. Lo negativo es que al fijarse en lo que gasta y no en lo que ingresa, la familia no revela si las familias consiguen evitar la pobreza recurriendo a elevados niveles de endeudamiento u otros mecanismos que entrañan una gran inseguridad financiera.

En lugar de utilizar variables monetarias algunos estudios sobre la pobreza utilizan indicadores directos del nivel de vida, tratando de identificar a aquellos individuos y familias que acumulan carencias y desventajas en una serie de áreas específicas (alimentación, vestido, vivienda, relaciones sociales, etc). Para ello se construyen índices de privación múltiple, que asignan a cada hogar una puntuación según la gravedad global de su situación en los campos considerados. Como principal desventaja de este enfoque tenemos la mayor dificultad para obtener este tipo de información y los problemas metodológicos ligados a su tratamiento: escoger los aspectos del nivel de vida que interesan, seleccionar indicadores válidos para cada área, eliminar la influencia de los gustos y estilos de vida, decidir la ponderación que se da a cada elemento y el método para agregar las distintas dimensiones de un valor que refleje la situación global, y determinar qué nivel de privación debe ser considerado crítico para identificar a la población pobre.

Además de elegir los parámetros de medida cualquier estudio sobre la pobreza debe decidir cuál es la unidad de análisis adecuada para analizar la situación económica de los individuos. Los estudios de pobreza suelen tomar como punto de partida las rentas del hogar o la unidad familiar, más que las rentas individuales, dado que los hogares toman decisiones económicas en común, comparten sus ingresos y realizan una serie de gastos que benefician por igual al conjunto de los miembros que los componen. 
No obstante, dado que los hogares tienen distinto tamaño y composición, la renta familiar total no es un buen criterio para comparar la situación económica de sus miembros .Tampoco suele utilizarse la renta per cápita del hogar, porque no tiene en cuenta las economías de escala asociadas a la vida conjunta.

En general, los estudios de pobreza y desigualdad hacen las comparaciones en términos de renta equivalente, resultado de dividir la renta del hogar entre una escala de equivalencia. Dichas escalas son coeficientes que dependen del tamaño y, a veces, también de las edades y otras características de los miembros del hogar. En concreto la escala de la OCDE, la de la OCDE modificada y las escalas paramétricas son algunas de las más utilizadas.

Un aspecto fundamental en la medición de la pobreza es la elección de un umbral o línea de pobreza, que debe servir para separar a la población pobre de la que no lo es. La elección del umbral dependerá del enfoque de la pobreza adoptado y del tipo de indicadores empleados. Los umbrales más comunes en los estudios actuales son los relativos, fijados como un determinado porcentaje de la media o mediada de la renta (o del gasto). Otros procedimientos menos extendidos son la elaboración de presupuestos mínimos para la cobertura de necesidades, las líneas subjetivas construidas a partir de las percepciones de la población o los umbrales políticos y legales.

Una vez identificada a la población pobre, hay que resumir el nivel de pobreza en determinadas sociedades, grupos o territorios, para lo cual se utilizan los índices de pobreza, que son medidas agregadas que permiten sintetizar la información relevante en una cifra que refleja la magnitud de la pobreza. El índice más habitual es la tasa de pobreza (personas pobres/población total) que mide la extensión o el gap de pobreza que se centra en la intensidad, es decir en las diferencias entre el nivel de vida de los pobres y el disfrutado por el resto de la sociedad (se calculan los desniveles individuales de pobreza o gap, es decir la renta que a cada individuo le falta para para llegar al umbral de la pobreza, luego se calcula la media de estos desniveles, y el resultado se expresa en términos de proporción respecto al umbral).

La necesidad de abordar el concepto de renta a microescala espacial, obliga a discutir la capacidad de llegar a una medida válida del concepto a través de variables alternativas.

Las que en el marco del proyecto Urbal se proponen al efecto, especialmente para la ciudad de Málaga, están orientadas a medir los ingresos de los individuos o los hogares. Las propuestas para su estudio son las siguientes:

- Ingresos o rentas de trabajo: 1. Ingresos de la población ocupada (cotizaciones de la SS en 11 categorías); 2. IRPF (Agencia Estatal de Administración Tributaria); 3.Prestaciones de desempleo (SPE) 
Igualmente podrían extrapolarse las rentas del trabajo a partir del conocimiento del sector de actividad y los salarios medios de dichos sectores.

- Ingresos empresariales: 1.Estimaciones indirectas a través del impuesto de IAE

IVA (AEAT); 2. Cotizaciones SS

- Transferencias sociales: 1.Pensiones contributivas gestionadas por el INSS (Incapacidad permanente, viudedad, Orfandad y Jubilación); 2. IRPF (AEAT); 3. Pensiones no contributivas gestionadas por la Consejería para la igualdad y el Bienestar social (Invalidez y Jubilación)

Cabría discutir el papel que puede concederse a otra información de la capacidad económica de los individuos, como es su patrimonio. Existen dos bienes, la vivienda y los vehículos, sobre los que es posible manejar información en formato de microdato, es decir, asociado a cada individuo. Las fuentes de información en el caso de España son para cada uno de ellos: 1. Impuesto de Bienes Inmuebles; 2. Impuesto de IVTM.

Otras Fuentes que podremos utilizar como referencia en el análisis de las variables: Panel de Hogares de la UE; Encuesta continua Presupuestos familiares; Encuesta Estructura salarial.

\subsubsection{Trabajo}

Sobre ocupación y actividad, la fuente más extensiva es el Censo de Población. Proporciona información sobre tres aspectos básicos:

$1^{\circ}$ Relación con la actividad. La población se clasifica en categorías tales como ocupado, parado que ha trabajado, parado busca su primer empleo (activos) e inactivos entre los que señalamos pensionista de jubilación, de viudedad, de orfandad, otras, rentista, labores del hogar, escolar.

$2^{\circ}$ Ocupación profesional: con la posible desagregación (a un dígito o dos dígitos) en diferentes niveles de detalle de acuerdo a codificaciones oficiales.

$3^{\circ}$ Situación profesional: empresario con empleados, empresario sin empleados, asalariado (fijo o eventual), ayuda familiar y miembro de cooperativas.

La información está disponible con diferenciación de género o edad, y accesible también con especificación para la persona de referencia del hogar. Puede ser explotada a nivel microespacial. 
La amplitud temática del censo se ve frustrada por su carácter estático. En el caso de España refleja la situación de 2001.

$\mathrm{Al}$ contrario del censo, el SPE constituye un registro vivo de la población activa

SPE: Información sobre población parada (edad, sector, duración) y tipos de contrato de la población ocupada

SS (Tesorería General): Altas de los trabajadores (tipo de trabajo, categoría profesional CNO y CNAE, tipo de contrato)

Como microdato o para microáreas la información del SPE o SS no es pública. La posibilidad de uso depende de los acuerdos que se alcancen en las distintas administraciones.

Como referencia para analizar las variables de este ámbito podemos utilizar la EPA que nos ofrece información muestral para la población parada por tiempo búsqueda empleo.

\subsubsection{Formación}

Dos fuentes relativas a la población que contemplan los caracteres de los individuos con relación al nivel educativo son el Censo y el Padrón. Ambas tienen dificultades importantes.

- El censo es una fuente que refleja la situación de la población para un momento dado. Es una instantánea del estado de una población. Como se ha dicho, en España, la situación reflejada es la de 2001. En concreto nos ofrece información sobre nivel de estudios de la población mayor de 16 años: Analfabetos, Sin estudios, Educación primaria, Educación secundaria, Educación Superior.

- El Padrón es un registro vivo de los habitantes en lo que se refiere a alta y bajas, pero los caracteres de cada individuo que se reflejaron en el momento de su registro, permanecen invariables hasta que se produzca un cambio residencial que de origen a una modificación del registro.

En consecuencia el Padrón es una fuente con graves limitaciones para estimar aspectos (como es el caso de los niveles educativos) que no son caracteres permanentes de los individuos.

Por su parte las Estadísticas Enseñanza en España (MECD), nos complementan esta información mostrando la Tasa de finalización de estudios por clase de enseñanza, los alumnos matriculados y la Tasa de escolarización 


\subsubsection{Salud}

La salud determina la calidad de vida, incide en la dependencia y adquiere perfiles diferentes en asociación con la pobreza.

El Anuario Estadístico de España, junto al Informe de Indicadores de Tratamiento, Urgencias y Mortalidad y el Boletín Epidemiológico semanal (Instituto Carlos III), nos informan agregadamente de las personas afectadas con enfermedades crónicas, incluida el SIDA y las drogodependencias. Estadísticas elaboradas las dos primeras a partir del trabajo del Instituto Nacional de Gestión Sanitaria y el Servicio Andaluz de Salud en el caso de España. El problema estriba en que la naturaleza de la información la hace poco proclive a su manejo como microdato.

Otros colectivos como las personas discapacitadas de más del 65\% de minusvalía quedan también registrados en el caso de España en la dependencia correspondiente de la Delegación de Consejería para la igualdad y el Bienestar social de las distintas comunidades autónomas. Las dificultades de acceso a la información a nivel de individuos (microdato) pueden ser similares.

La confidencialidad no es trasgredida si se trata de datos agregados, aunque hay que tener presente que para explotar la información por unidades de trabajo social (el horizonte es un SIG) en alguna fase la persona enferma o discapacitada ha de ser identificada por su dirección o su DNI.

En ambos casos la disponibilidad de la información está supeditada a los acuerdos que puedan establecerse entre los organismos públicos.

De manera complementaria y como ya hemos enumerado en el caso de otras dimensiones se puede utilizar le información relativa a la Encuesta sobre discapacidades, deficiencias y estado de salud y la Encuesta Nacional de Salud elaborada por el INE.

Otra base de información la aportan los propios servicios municipales, relativa únicamente a las personas que demandan prestaciones en función de estos problemas. La base de datos de estos servicios (SIUSS, RIM, empresa MasCerca) aun no siendo una medición de toda la población puede ser muy significativa.

\subsubsection{Vivienda y entorno ambiental}

Las condiciones de habitabilidad se convierten en muchas ocasiones en una consecuencia de las precarias o difíciles condiciones de vida, pero igualmente las retroalimentan, de manera que se pueden convertir en mecanismos para detectar la existencia de vulnerabilidad social

Para cubrir este campo podemos recurrir a las siguientes fuentes: 
- Censo de viviendas 2001 que cuenta con información sobre el régimen de tenencia, características y dotaciones de viviendas y edificios.

- IBI: reúne información sobre las dimensiones y antigüedad de las viviendas

- Registro de las viviendas en régimen de alquiler del Instituto Municipal de la vivienda.

Igualmente contamos con la información utilizada como Diagnóstico en áreas especialmente difíciles de la ciudad para las que el Área de bienestar social ha elaborado Planes Especiales de acción, que pensamos podemos complementar con la información que tiene la Dirección General de la policía nacional (criminalidad).

\subsubsection{Vulnerabilidad en función de la familia o el hogar}

Entenderíamos como tal el conjunto amplio de problemas que hacen del núcleo familiar, por su inexistencia o por su problemática, un factor negativo en el desarrollo del individuo. Pudiéndose definir varios ámbitos o subdimensiones:

1. La inexistencia de un núcleo familiar: soledad y enfermedad; soledad y ancianidad; inmigrantes pendientes de reunificación familiar.

2. Inexistencia de un hogar de referencia: personas sin techo.

3. Estructuras del hogar que denotan cierta vulnerabilidad: tasa inusual de personas dependientes respecto a personas con ingresos; acumulación de personas adultas en situación de paro; combinación de enfermedad y dependencia de menores; miembros del hogar convictos o exconvictos.

4. Situaciones que desvelan conflictos del entorno familiar: malos tratos a menores; violencia de género; violencia de familia; absentismo escolar.

5. Miembros del hogar con problemas de marginación: delincuencia; drogodependencia; prostitución .

Las fuentes documentales oficiales proporcionan diferente cobertura para los distintos aspectos señalados.

- Censo de Población. Hogares por total de personas, número de parados y ocupados.

- EPA (como fuente de referencia). Viviendas familiares por números de personas, activos, ocupados y parados; y según la situación respecto a la actividad de la persona de referencia 
- Padrón. Información sobre los miembros de los hogares y las familias: número personas en la vivienda y familia, procedencia, edad

- SS: Situación afiliados extranjeros

- Sobre personas drogodependientes, con Sida y discapacitadas, las fuentes ya señaladas en el ámbito de la Salud

- Sobre absentismo escolar la información de la Delegación Consejería de Educación y Ciencia.

- Ministerio Interior. Detenidos, Población reclusa, Violencia familiar y de género; extranjeros solicitantes de asilo

- Encuesta sobre las personas sin hogar e Información recogida por los Servicios Ayuntamiento se constituirían en una alternativa para aspectos no abarcados por la documentación extensiva.

\subsubsection{Participación ciudadana}

Se trata de una información que sólo podríamos conocer a nivel de encuesta y mediante los datos de los Centros de Servicios Municipales

\subsection{Propuesta de indicadores sociales}

Conscientes de que no todos los indicadores expuestos en los apartados anteriores pueden ser utilizados en el estudio de la realidad social de nuestras ciudades, en los diversos proyectos que se realicen, sin embargo la utilización de un Sistemas de Información Geográfica hace viable que este amplio conjunto de información sea recogida o al menos esté presente en los organigramas de las instituciones adecuadas, y además que esté disponible para su elaboración de cara a las posibles nuevas situaciones y demanda que se produzcan en el futuro

La propuesta de indicadores sociales que se presentó en el seminario llevado a cabo en Málaga en septiembre de 2005 fue amplia y versátil (Cuadro 4), por lo que no definitiva. Se trataba de una extensa relación o listado con la que los socios podían trabajar de acuerdo con sus objetivos y posibilidades y sobre todo en relación con las fuentes disponibles en un nivel de desagregación microespacial y con la mayor actualización posible. A partir de esta amplia propuesta se pudo determinar un conjunto de indicadores mínimos comunes a todos los socios, con el fin de que el sistema que se ponga en marcha sea operativo por sus posibilidades de comparabilidad.

De la propuesta de indicadores sociales se llegó a consensuar 22 indicadores comunes para todos los socios, de ellos, como aparece en el cuadro 4 han sido los denominados indicadores de contexto los más fácilmente asumibles por el conjunto de ciudades participantes, pues de los 15 propuestos, las 
2/3 partes son realizables por la totalidad de los socios; en segunda posición se sitúan los indicadores de formación que de nueve 4 son comunes para los 7 socios presentes en el proyecto; en tercer lugar están los indicadores de trabajo, de los que sólo son comunes 4 de trece. Los indicadores menos asumibles por los socios han sido los de nivel de ingresos, de cuatro sólo uno lo puede calcular todos los socios, los de calidad de la vivienda, que de cinco, sólo son comunes dos, y por último hay que señalar dos conjunto de indicadores en los que no hay ningún indicador asumible a la vez por todos los socios, estos son los indicadores sobre acceso a la salud y los indicadores sobre la vulnerabilidad familiar

Por ciudades, ha sido la municipalidad de El Bosque (Chile) la que se sitúa en primera posición al poder llevar a cabo un total de 36 indicadores, seguida de las ciudades de Málaga y Granada (España) con 27 cada una de ella, en tercera posición se encuentran las ciudades peruanas de Lima y Oyón (26 indicadores) y por último las de Feira de Santana, Aserrí y Navolato que pueden asumir la realización de 23 indicadores cada una de ellas. 


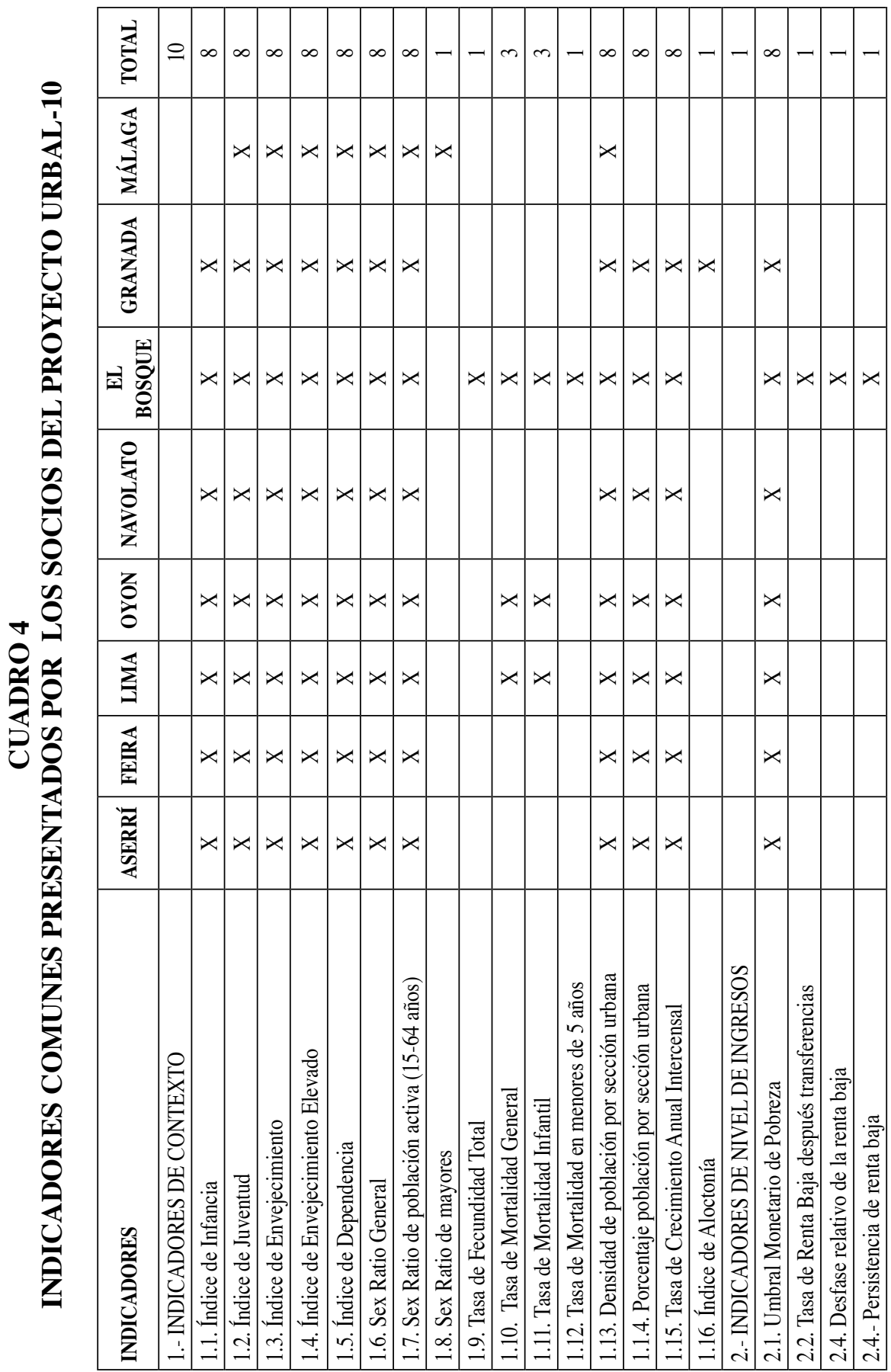


Indicadores para medir situaciones de vulnerabilidad...

\begin{tabular}{|c|c|c|c|c|c|c|c|c|c|c|c|c|c|c|c|c|c|c|c|c|c|c|c|c|}
\hline 言 & in & $\infty$ & $\infty$ & $\infty$ & $\infty$ & -1 & $\infty$ & 0 & $m$ & 0 & 0 & 0 & 0 & $0 \mid+$ & $\infty$ & $\infty$ & $\infty$ & $\infty$ & 0 & 0 & 0 & 0 & 0 & $\sim$ \\
\hline 忑 & & $x$ & & $x$ & & $凶$ & & $x$ & $x$ & & & & & & $x$ & & & & & & & & & \\
\hline $\begin{array}{l}\text { 岕 } \\
\text { 离 }\end{array}$ & & $x$ & $x$ & $x$ & 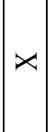 & & $x$ & & $x \mid$ & & & & & & $x$ & $\rtimes \mid$ & $x$ & $x$ & & & & & & \\
\hline 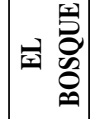 & & $\times 1$ & $x$ & $x$ & $x$ & $\rtimes$ & $x$ & & $x$ & & & & & & $x$ & $\rtimes$ & $x$ & $x$ & & & & & & \\
\hline 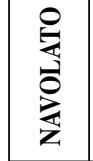 & & $x$ & $x$ & $x$ & $x$ & & $x$ & & & & & & & & $x \mid$ & $\Varangle \mid$ & $x$ & $x$ & & & & & & \\
\hline 元 & & $x$ & $x$ & $x$ & $x$ & & $x$ & & & & & & & & $x \mid$ & $\rtimes$ & $x$ & $x$ & & & & & & \\
\hline 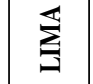 & & $x \mid$ & $x$ & $x$ & $x$ & & $x$ & & & & & & & & $x$ &  & $x$ & $x$ & & & & & & \\
\hline 丞 & & $x$ & $x$ & $x$ & $x$ & &  & & & & & & & & $x \mid$ & $x \mid$ & $x$ & $x$ & & & & & & \\
\hline 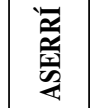 & & $\rtimes$ & $x$ &  & $x$ & &  & & & & & & & & $x$ & $\rtimes$ & $x$ & $x$ & & & & & & \\
\hline 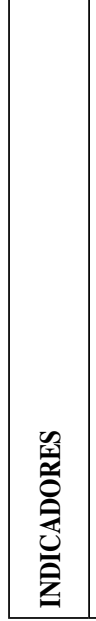 & 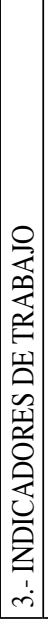 &  & 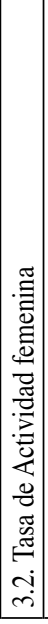 & 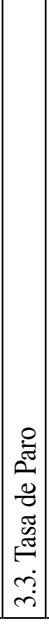 & 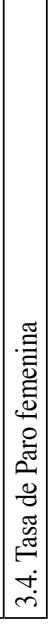 & 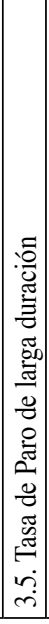 &  & 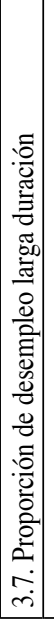 &  & 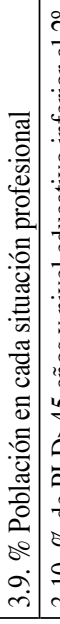 &  & 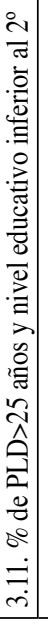 &  & 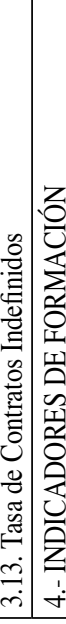 &  & 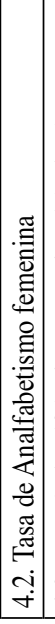 & 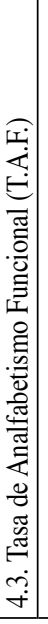 & 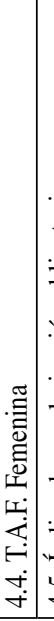 & 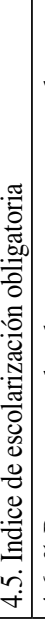 & 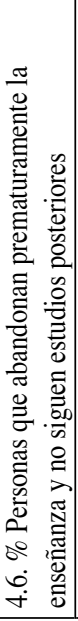 & 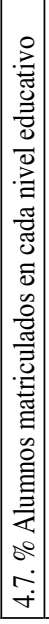 & 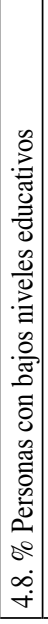 & 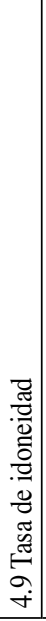 & 盖 \\
\hline
\end{tabular}




\section{BIBLIOGRAFÍA}

BAUER, R. (1966) (Ed): Social Indicators, Cambridge, MTI, Press.

CARMONA GUILLÉN, J.A, (1977): Los indicadores sociales hoy. Centro de Investigaciones sociológicas, Colección Monografías, $\mathrm{n}^{\circ} 2$, Madrid.

CASAS F (1989): Técnicas de investigación social: los indicadores sociales y psicosociales: Teoría y práctica, Barcelona. Promociones y Publicaciones Universitarias.

COMITÉ DE PROTECCIÓN SOCIAL (2001): Informe sobre los indicadores en el ámbito de la pobreza y la exclusión social, Bruselas

GIMENO ULLASTRE, J.A, (Coord) (2004): Exclusión social y estado del bienestar, Ed. Centro de Estudios Ramón Areces y Fundación Luis Vives, Madrid

INE (1993): Indicadores sociales, Ed INE, Madrid.

OCDE (2002): Panorama de la Sociedad. Los indicadores sociales de la OCDE, OCDE, $2002 \mathrm{http}: / / \mathrm{www}$.oece.org/els/social.

PENA TRAPERO, J.B. (1977): Problemas de la medición del bienestar y conceptos afines (Una aplicación al caso español), INE. Madrid.

SUBIRATS JOAN (DIR) : Pobreza y exclusión social. Un análisis de la realidad española y europea, Fundación la Caixa. www.estudios.lacaixa.es

ZARZOSA ESPINA, P. (1996): Aproximación a la medición del Bienestar Social, Universidad de Valladolid, Serie Economía no 23. 